\title{
Archivos \\ de Cardiología de Méxíco

\section{EI SPRINT en la práctica médica: ¿es momento de modificar el manejo de la hipertensión arterial en Latinoamérica?}

\author{
José Kelvin Galvez-Olortegui ${ }^{\mathrm{a}, \mathrm{b}, \mathrm{c}, \mathrm{d}, *}$, Yudy Condor-Rojas ${ }^{\mathrm{a}, \mathrm{e}}$, \\ Tomas Vladimir Galvez-Olortegui ${ }^{\mathrm{a}, \mathrm{f}}$ y Luis Camacho-Saavedra ${ }^{\mathrm{a}, \mathrm{d}, \mathrm{g}}$
}

\author{
a Scientia Clinical and Epidemiological Research Institute, Trujillo, Perú \\ ${ }^{\text {b } C e n t r o ~ M e ́ d i c o ~-~ 32 . ~}{ }^{\circ}$ Brigada de Infantería-Ejército del Perú, Trujillo, Perú \\ c Guidelines International Network (G-I-N), Escocia \\ d Facultad de Medicina, Universidad Nacional de Trujillo, Trujillo, Perú \\ e Facultad de Medicina, Universidad Peruana de Ciencias Aplicadas, Lima, Perú \\ ${ }^{f}$ Escuela de Posgrado, Universidad Privada Antenor Orrego, Trujillo, Perú \\ g Servicio de Medicina Interna, Hospital I «Florencia de Mora», Trujillo, Perú
}

Recibido el 14 de enero de 2016; aceptado el 17 de junio de 2016

\section{PALABRAS CLAVE \\ Hipertensión; \\ Presión arterial; \\ Guías de práctica \\ clínica; \\ Ensayo clínico; \\ Perú}

\begin{abstract}
Resumen El presente documento analiza la factibilidad de la implementación de los resultados del ensayo clínico SPRINT, la necesidad de replantear las guías de práctica clínica (GPC) para el manejo de la hipertensión arterial y los costos asociados a su aplicabilidad en la práctica diaria. El SPRINT es un ensayo clínico que comparó el control intensivo de la presión arterial sistólica $<120 \mathrm{mmHg}$ y $<140 \mathrm{mmHg}$ sobre las complicaciones cardiovasculares, generando un gran impacto en el mundo seguido de la publicación de diversos estudios que han abordado desde diversas perspectivas la relevancia, utilidad, aplicabilidad y aspectos controversiales del SPRINT. El logro de la presión arterial meta es uno de los temas más discutidos en las GPC de hipertensión arterial de mayor uso a nivel mundial y latinoamericano. El SPRINT ha generado y generará un gran impacto en las GPC, siendo necesaria la reevaluación de las presiones arteriales meta y su inclusión en las GPC futuras, como ha sido considerado por la guía canadiense 2016 y será considerado en la actualización de la Guía NICE programada para junio.

El ensayo SPRINT plantea nuevas evidencias para el manejo de la hipertensión arterial, útil en personas mayores de 50 años, procedentes de poblaciones urbanas, con riesgo cardiovascular definido y sin comorbilidades asociadas. La aplicabilidad del SPRINT en Latinoamérica es limitada por el aumento de costos asociados a la atención de salud integral del paciente hipertenso, la baja cobertura de atención y escasez de programas integrales de atención.

(c) 2016 Instituto Nacional de Cardiología Ignacio Chávez. Publicado por Masson Doyma México S.A. Este es un artículo Open Access bajo la licencia CC BY-NC-ND (http://creativecommons. org/licenses/by-nc-nd/4.0/).
\end{abstract}

\footnotetext{
* Autor para correspondencia: Mz. G Lt 22 Urb. Vista Hermosa, Trujillo, Perú.
} Correo electrónico: jgalvezo@scientiaceri.com (J.K. Galvez-Olortegui). 


\section{KEYWORDS}

Hypertension; Arterial Pressure; Practice guidelines; Clinical trial;

Perú
SPRINT on clinical practice: It's time to change the management of arterial hypertension in Latin America?

Abstract This paper analyzes the feasibility of the implementation of SPRINT trial results, the need to rethink the clinical practice guidelines(CPG) for the management of arterial hypertension and associated costs with daily practice applicability. SPRINT is a clinical trial comparing systolic blood pressure control $<120 \mathrm{mmHg}$ and $<140 \mathrm{mmHg}$ over cardiovascular complications, generating a great worldwide impact followed by publication of several studies that addressed relevance, usefulness, applicability and controversial aspects of SPRINT from different perspectives. Achieving blood pressure goals is one of the most discussed issue in widely used hypertension CPG around the world and in Latin American. SPRINT has generated and will generate a great impact on CPG, being necessary the reassessment of blood pressure goals and inclusion in future CPG, as has been considered in 2016 Canadian guideline and will be considered in NICE guideline update scheduled for June.

The SPRINT trial raises new evidence for the management of hypertension, useful in people over 50 years, from urban populations, with defined cardiovascular risk without associated comorbidities. The applicability of SPRINT in Latin America is limited by increased costs associated with hypertensive patients' integrated health care, low care coverage, and lack of integrated care programs.

(C) 2016 Instituto Nacional de Cardiología Ignacio Chávez. Published by Masson Doyma México S.A. This is an open access article under the CC BY-NC-ND license (http://creativecommons. org/licenses/by-nc-nd/4.0/).

\section{Introducción}

La medicina basada en evidencias plantea que cada acto realizado en la práctica médica debe estar respaldado por estudios científicos, y estos a su vez deben mejorar la evidencia disponible. Tomando en cuenta la pirámide de la EBM desarrollada por DiCenso ${ }^{1}$, los estudios secundarios, tales como las revisiones sistemáticas, estudios de síntesis y las guías de práctica clínica (GPC), constituyen el mayor nivel de evidencia y destacan por su utilidad en la práctica médica diaria dado que son «un conjunto de recomendaciones basadas en una revisión sistemática de la evidencia y en la evaluación de los riesgos y beneficios de las diferentes alternativas, con el objetivo de optimizar la atención sanitaria a los pacientes» ${ }^{2}$.

Toda GPC debe responder a las prioridades sanitarias y a la disponibilidad técnica, logística y financiera de cada país ${ }^{3}$; por lo tanto, al momento de implementar sus recomendaciones deben considerar factores facilitadores y limitantes de sus sistemas de salud, para mejorar la adherencia por parte del personal de salud, de manera efectiva y eficiente.

En los últimos años, tanto la adaptación, como la adopción de GPC adquieren mayor relevancia en Latinoamérica, debido a la reducción de los costos relacionados frente a la elaboración de las mismas y a la mejor comprensión de los beneficios de la implementación de una GPC.

El presente documento analiza la factibilidad de la implementación de los resultados del ensayo clínico SPRINT ${ }^{4}$, reflexionando sobre la necesidad de replantear las GPC para el manejo de la hipertensión arterial (HTA) y los costos asociados a su aplicabilidad en la práctica diaria.

\section{Ensayo clínico SPRINT}

El SPRINT es un ensayo clínico, desarrollado en pacientes hipertensos mayores de 50 años con alto riesgo de eventos cardiovasculares y una presión arterial (PA) entre 130 a $180 \mathrm{mmHg}$. Su hipótesis propone que la PA sistólica (PAS) menor de $120 \mathrm{mmHg}$ disminuye la tasa de complicaciones cardiovasculares comparado con una PAS menor de $140 \mathrm{mmHg}$ y analiza si esta reducción de los niveles de PAS aportan beneficio. El estudio excluye a pacientes con diabetes mellitus, ictus previo, poliquistosis renal, insuficiencia cardiaca, consumo frecuente de alcohol e historia de poca adherencia al tratamiento. Las diferencias de complicaciones entre ambos grupos se determinaron mediante el análisis de sobrevida de Kaplan Meier y hazard ratios, con una reducción de riesgo del 25\%; y adicionalmente evaluó la reducción de riesgo en otros desenlaces. El ensayo plantea que la diferencia en el desenlace renal de ambos grupos, puede estar relacionado con el efecto hemodinámico reversible a nivel intrarrenal, ocasionado por la mayor reducción en la presión sanguínea y el mayor uso de diuréticos, inhibidores de la enzima convertidora de angiotensina y bloqueadores de receptores de angiotensina en el grupo de tratamiento intensivo.

En este contexto, podemos observar que:

- La reducción de riesgo absoluto y porcentual no muestra resultados similares.

- El número necesario a tratar planteado es alto (61 para el desenlace primario y 90 para muertes por causas cardiovasculares).

- El número promedio de medicamentos utilizados por grupo no difiere en gran medida; aunque aproximadamente el $25 \%$ de los pacientes con tratamiento intensivo utilizaron 4 o más medicamentos.

- No se realizó un análisis estratificado para los participantes con 4 o más medicamentos (hipertensión resistente).

- El 86.6 y $68.9 \%$ de pacientes con tratamiento intensivo y estándar respectivamente, requirió dos o más medicamentos (promedio de medicamentos 2.7 contra 1.8 ). 
- El grupo con tratamiento intensivo presentó más efectos adversos a nivel global (4.7\% contra $2.5 \%$ ) y según tipo de evento.

- El uso de 3 o 4 medicamentos y la presencia de eventos adversos se asocia a un incremento de costos.

- No se ha medido la frecuencia de accidentes cerebrovasculares.

- El estudio no alcanzó la PA meta menor a 120 mmHg (meta lograda: $121.5 \mathrm{mmHg}$ ).

- Una diferencia de $1.5 \mathrm{mmHg}$ se acompaña de cambios fisiológicos que pudieron modificar el desenlace esperado y los eventos adversos.

\section{Impacto del SPRINT: control intensivo de la presión arterial}

Desde el anuncio de los resultados preliminares (11 de septiembre $)^{5}$, la publicación en línea (09 de noviembre) y oficial ( 26 de noviembre) ${ }^{4}$, el SPRINT ha generado un gran impacto en el mundo publicándose editoriales ${ }^{6-10}$, comentarios, revisiones sistemáticas/metaanálisis ${ }^{11}$, estudios transversales ${ }^{12}$, análisis secundarios de estudios previos (ACCOMPLISH) ${ }^{13}$, e incluso evaluándose la detención/continuación del ensayo clínico en ejecución ESH$\mathrm{CHL}_{-} \mathrm{SHOT}^{14}$. Estos estudios han abordado desde diversas perspectivas la relevancia, utilidad, aplicabilidad y aspectos controversiales del SPRINT, relacionados con la PA meta ideal o alcanzada, los efectos adversos más frecuentes (hipotensión, síncope, hiponatremia, hipopotasemia, etc. $)^{6,8}$, la aplicabilidad a pacientes con comorbilidades (pacientes diabéticos, nefrópatas), el manejo de la HTA de difícil control $\mathrm{y} / 0$ resistente ${ }^{6}$, la caída de la PAS ${ }^{9}$, el rol de la curva $\mathrm{J}^{7,10}$ y de la PA diastólica ${ }^{8}$.

Previamente, el ensayo clínico ACCORD, realizado en pacientes diabéticos, comparó las $\mathrm{PA}<120 \mathrm{mmHg}$ y $\mathrm{PA}<140 \mathrm{mmHg}$, no reportando diferencia significativa en los eventos cardiovasculares ${ }^{15}$. Revisiones sistemáticas posteriores que incluyeron al ACCORD y otros estudios, reportaron una reducción del riesgo cardiovascular mayor y de algunas complicaciones en personas de alto riesgo cardiovascular (diabéticos y nefrópatas), en el grupo con PA $<120 \mathrm{mmHg}^{16}$. El SPRINT planteó un objetivo similar al del ACCORD, pero reclutó el doble de participantes, con una edad promedio mayor (68/62 años), incluyendo a los pacientes con enfermedad renal crónica y excluyendo a los pacientes diabéticos ${ }^{17}$.

Estudios publicados posteriores al SPRINT han mostrado el beneficio del control intensivo de la PA, y un análisis secundario del ACCOMPLISH ${ }^{18}$ que evaluó el efecto de la PAS $(<120,<130$ y $<140 \mathrm{mmHg})$ sobre el desenlace cardiovascular, reportó un efecto beneficioso en pacientes con y sin diabetes ${ }^{13}$. Dos metaanálisis posteriores, han reportado un efecto positivo del control intensivo de la PA, a un nivel claro menor de $130^{11,19}$; aunque los niveles menores a 120 se mantienen en controversia. Pese a los resultados relevantes del SPRINT, es necesario considerar que las nuevas PA meta planteadas por el estudio mostraron preferentemente una reducción de la mortalidad global y de la insuficiencia cardiaca; sin embargo como todo ensayo clínico, sus resultados son limitados por su validez externa, al ser aplicables en poblaciones urbanas, sin diabetes y no pudiendo ser inmediatamente generalizables a toda la población de un país ${ }^{20,21}$, especialmente a los países latinoamericanos.

\section{Aplicabilidad del SPRINT en Latinoamérica: guías de práctica clínica y costos asociados}

La prevalencia de HTA en Latinoamérica es en promedio de $20.2 \%$ en la población general ${ }^{22}$, en el Perú es entre el 19.2 y $23.7 \%{ }^{23,24}$, en Argentina de $45.4 \%$, en Chile de $39.9 \%$, en Uruguay de $44.1 \%{ }^{24}$ y de $18 \%$ en México $^{25}$. La elevada prevalencia de la HTA en Latinoamérica obliga a la necesidad de contar con GPC basadas en evidencia, que se actualicen paralelamente a la velocidad de producción de nueva evidencia científica, por lo menos 2 o 3 años después de su publicación ${ }^{26}$, y reevaluadas en un período no mayor de 3 años ${ }^{27}$; situación que redundará en una mejor calidad de atención y disminución de los costos asociados a ella.

El logro de la PA meta es uno de los temas más discutidos en las GPC de HTA de mayor uso a nivel mundial y latinoamericano ${ }^{28}$, la mayoría de las cuales fueron publi-

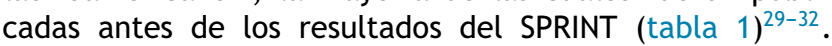
Respecto a las metas de PA, no existen cambios en las guías previas de la Sociedad Europea ${ }^{33}$ ni en la Canadiense ${ }^{34}$, pero sí entre el octavo y séptimo reporte (PA meta: $140 / 90 \mathrm{mmHg})^{35}$. A nivel de Latinoamérica, las GPC disponibles presentan un alcance local y son escasamente difundidas a través de revistas internacionales ${ }^{36}$, destacando dos guías por haber incluido estudios regionales (tabla $1)^{37,38}$. Estas recomendaciones sustentadas en gran medida en las GPC internacionales previamente mencionadas, no han sido realizadas por grado de recomendación y nivel de evidencia; afectando su utilidad por los profesionales de la salud $^{36}$, pues las implicancias de un alto nivel de evidencia/grado de recomendación permitirán una aplicación más amplia de esta ${ }^{39}$ y una mejor adherencia.

El SPRINT ha generado y generará un gran impacto en las GPC, siendo necesaria la reevaluación de las PA meta y su inclusión en las GPC futuras ${ }^{40}$, como ha sido considerado por la guía canadiense $2016^{32,41}$ y será considerado en la actualización de la guía del National Institute for Health and Care Excellence (NICE - 2011) ${ }^{42}$ programada para junio del 2016.

Una de las mayores limitaciones de la aplicabilidad del SPRINT en Latinoamérica es el uso de equipos para la medición de la PA, dado que en los países latinoamericanos aún es frecuente el uso del esfigmomanómetro de mercurio o aéreo ${ }^{24}$. En el SPRINT, se realizaron 3 mediciones a los pacientes sentados, después de cinco minutos de reposo antes de la medición, sin conversaciones durante el periodo de reposo o medición; condiciones difícilmente alcanzables por el personal de salud en Latinoamérica. La medición sin estas condiciones podría generar una sobrestimación de la PA conduciendo a un tratamiento más complejo, con más medicamentos y acompañado de mayores efectos adversos y costos incrementados ${ }^{17}$.

Adicionalmente, las altas prevalencias implican inevitablemente un aumento anual de costos directos e indirectos ${ }^{43}$ relacionados con la prevención, diagnóstico y tratamiento, así como al manejo de sus secuelas y comorbilidades asociadas; consecuencia de la nefropatía relacionada, el infarto 
Tabla 1 Presiones arterial meta en las GPC internacionales

\begin{tabular}{|c|c|c|c|c|c|}
\hline & Octavo reporte ${ }^{29}$ & $\begin{array}{l}\text { Guía de la Sociedad } \\
\text { Europea de Hipertensión } \\
30\end{array}$ & $\begin{array}{l}\text { Programa canadiense para } \\
\text { la educación en } \\
\text { hipertensión }^{31,32}\end{array}$ & $\begin{array}{l}\text { Guía latinoamericana } \\
\text { de hipertensión } \\
37\end{array}$ & $\begin{array}{l}\text { Consenso latinoamericano } \\
\text { de hipertensión en pacientes } \\
\text { con diabetes tipo } 2 \text { y síndrome } \\
\text { metabólico }\end{array}$ \\
\hline País(año) & Estados Unidos (2014) & Europa (2013) & Canadá (2015 y 2016) & Latinoamérica (2009) & Latinoamérica (2013) \\
\hline \multirow[t]{3}{*}{$\begin{array}{l}\text { Presión } \\
\text { arterial meta }\end{array}$} & $\begin{array}{l}150 / 90 \mathrm{mmHg} \text { (pacientes } \\
\text { de } 60 \text { años o más) } \\
\text { (recomendación } \\
\text { fuerte-grado A) }\end{array}$ & $\begin{array}{l}<140 / 90 \mathrm{mmHg} \text { (pacientes } \\
\text { de riesgo bajo o } \\
\text { moderado) (nivel de } \\
\text { recomendación I, clase B) }\end{array}$ & $\begin{array}{l}<140 / 90 \mathrm{mmHg} \\
(\text { recomendación grado } \mathrm{A})^{*}\end{array}$ & $<140 / 90 \mathrm{mmHg}$ & $<130 / 80 \mathrm{mmHg}$ \\
\hline & $\begin{array}{l}\text { Si la meta alcanzada es } \\
\text { menor y no se presentan } \\
\text { efectos adversos, el } \\
\text { tratamiento no necesita } \\
\text { ser ajustado }\end{array}$ & $\begin{array}{l}<130 / 90 \text { (pacientes con } \\
\text { comorbilidades) }\end{array}$ & $\begin{array}{l}\text { Expectantes hasta } \\
<150 \mathrm{mmHg}(\geq 80 \text { años) } \\
\text { (recomendación C) }\end{array}$ & & \\
\hline & & & $\begin{array}{l}<120 \mathrm{mmHg} \text { (pacientes } \\
\text { en alto riesgo, más } \\
\text { de } 50 \text { años, } \mathrm{PA} \geq 130 \text { ) }\end{array}$ & & \\
\hline
\end{tabular}


de miocardio no fatal, y el número de medicamentos utilizados $^{43}$; con un costo aproximado per cápita de \$21 en Latinoamérica y $\$ 362$ en países desarrollados ${ }^{44}$. En Perú, el costo anual total para HTA es superior al presupuestado $(114 \%)^{45}$, generando una gran limitación en su manejo. Por otro lado, la cobertura de atención de hipertensos en países latinoamericanos es baja, debido a que el acceso y la oportunidad de atención de salud son limitados, con marcadas dificultades en la implementación de programas de atención integral de la HTA; así como el manejo de sus secuelas y rehabilitación asociada. El incremento de costos de estas nuevas recomendaciones para el manejo de la hipertensión, afectarán también la cobertura de servicios de cualquier seguro de salud, sea público, privado o mixto.

Del mismo modo, es conocido el actual desabastecimiento de las farmacias del sector público de la mayoría de los países latinoamericanos, que afecta la factibilidad de la implementación de las recomendaciones del SPRINT a nivel poblacional, principalmente porque lograr PA meta más bajas $(120 \mathrm{mmHg})$ requiere de 3 a 4 medicamentos, que al no ser atendidas por los gobiernos nacionales, redundará en mayores costos para el paciente. Esta situación se agrava cuando analizamos los petitorios de medicamentos en el sector público, y detectamos que no incluyen medicamentos genéricos con combinaciones fijas y que los disponibles en el mercado privado son altamente costosos para ser asumidos por la población de bajos recursos, que constituyen el mayor porcentaje en los países latinoamericanos. Según el observatorio de medicamentos, en el Perú el costo mínimo para un tratamiento con medicamentos separados de losartán $50 \mathrm{mg}$ BID/hidroclorotiazida $25 \mathrm{mg}$ QD es de $\$ 1.72$ al mes, comparado con la combinación fija de $\$ 22.7^{46}$; mientras que el uso de dosis de combinación fijas como el amlodipino $10 \mathrm{mg} /$ valsartan $160 \mathrm{mg} / \mathrm{HCT} 12.5 \mathrm{mg}$ podría llegar hasta $\$ 74.5$ al mes ${ }^{46}$. En México, el costo del tratamiento con medicamentos separados de losartán/hidroclorotiazida puede ser superiores a $\$ 19.6^{47}$.

Los medicamentos de combinación a dosis fijas permiten una mejor adherencia y eficacia en tratamiento antihipertensivo ${ }^{48}$, sin embargo no se encuentran disponibles en los servicios de salud en Latinoamérica, y los altos costos de estos pueden limitar la adherencia al tratamiento por los pacientes de escasos recursos; mucho más teniendo en cuenta que la posibilidad de cobertura de los antihipertensivos por el paciente dependerá del salario mensual recibido $^{49}$. Otro aspecto, relacionado con la polifarmacia, son los costos de las atenciones por los efectos adversos o secundarios, que conduce de manera inevitable al fracaso o abandono terapéutico, a causa de la inadecuada adherencia de los pacientes y del personal de salud al tratamiento ${ }^{50}$, fomentando mayores inequidades en los países latinoamericanos.

Es preciso mencionar que independientemente del tiempo de enfermedad y los determinantes sociales relacionados, la eficiencia de un adecuado control de la HTA se ve reflejado positivamente en la disminución de la carga de enfermedad y por ende la morbimortalidad de los pacientes, las cuales se asocian a los costos familiares y sociales correspondientes. En tal sentido consideramos la necesidad de realizar estudios de costo beneficio en relación con los años de vida perdidos ajustados a salud, como los realizados en Chile $\mathrm{e}^{51}$, y sustentados en función de la GPC utilizada ${ }^{52}$.

\section{Conclusiones}

El ensayo SPRINT plantea nuevas evidencias para el manejo de la HTA, que han mostrado su utilidad en personas mayores de 50 años, procedentes de poblaciones urbanas, con riesgo cardiovascular definido y sin comorbilidades asociadas, no siendo aplicable a todos los pacientes con HTA.

La aplicabilidad del SPRINT en Latinoamérica es limitada por el aumento de costos asociados a la atención de salud integral del paciente hipertenso, la baja cobertura de atención y escasez de programas integrales de atención de los pacientes hipertensos en los países latinoamericanos, el desabastecimiento de las farmacias del sector público, los altos costos de los medicamentos con combinaciones fijas, y la inequidad en salud que generarán altos costos del tratamiento asumido por los pacientes.

\section{Recomendaciones}

Completar las recomendaciones del SPRINT con estudios de costo beneficio en relación con los años de vida perdidos ajustados a salud, considerando los determinantes sociales claves como el nivel educativo y sociocultural de los pacientes, entre otros.

Desarrollar un plan de fortalecimiento de la atención primaria de la salud para el abordaje de la HTA.

\section{Financiación}

Ninguna.

\section{Conflicto de intereses}

Los autores declaran no tener ningún conflicto de intereses.

\section{Bibliografía}

1. Dicenso A, Bayley L, Haynes RB. Accessing preappraised evidence: Fine-tuning the $5 \mathrm{~S}$ model into a $6 \mathrm{~S}$ model. Ann Intern Med. 2009;151:5-6.

2. Institute of Medicine. Clinical practice guidelines we can trust. Washington DC: The National Academies Press; 2011.

3. Oxman AD, Schünemann HJ, Fretheim A. Improving the use of research evidence in guideline development: 2 . Priority setting. Health Res Policy Syst. 2006;4:1-7.

4. The SPRINT Research Group. A Randomized trial of intensive versus standard blood-pressure control. NEJM. 2015;373:2103-16.

5. National Hearth, Lung and BI. Landmark NIH study shows intensive blood pressure management may save lives [Internet]. Estados Unidos:www.nhlbi.nih.gov [consultado $25 \mathrm{Abr}$ 2016]. Disponible en: http://www.nhlbi.nih.gov/news/pressreleases/2015/landmark-nih-study-shows-intensive-bloodpressure-management-may-save-lives

6. Ruilope LM, Banegas JR. El estudio SPRINT en la práctica clínica: ¿se debe modificar el objetivo de control de la presión arterial? Hipertens Riesgo Vasc. 2016;33:47-50.

7. Kjeldsen SE, Oparil S, Narkiewicz K, et al. The J-curve phenomenon revisited again: SPRINT outcomes favor target systolic blood pressure below $120 \mathrm{mmHg}$. Blood Press. 2016;25:1-3.

8. Touyz RM, Dominiczak AF. Successes of SPRINT, but still some hurdles to cross. Hypertension. 2016;67:268-9. 
9. Esler M. SPRINT, or false start, toward a lower universal treated blood pressure target in hypertension. Hypertension. 2016;67:266-7.

10. Jones DW, Weatherly L, Hall JE. SPRINT. What remains unanswered and where do we go from here? Hypertension. 2016;67:261-2.

11. Thomopoulos C, Parati G, Zanchetti A. Effects of blood pressure lowering on outcome incidence in hypertension: 7. Effects of more vs. less intensive blood pressure lowering and different achieved blood pressure levels - updated overview and metaanalyses of randomized trials. J Hypertens. 2016;34:613-22.

12. Bress AP, Tanner RM, Hess R, et al. Generalizability of SPRINT results to the U.S. adult population. J Am Coll Cardiol. 2016;67:463-72.

13. Weber MA, Bloch M, Bakris GL, et al. Cardiovascular outcomes according to systolic blood pressure in patients with and without diabetes: an ACCOMPLISH substudy. J Clin Hypertens. 2016;18:299-307.

14. Zanchetti A, Liu L, Mancia G, et al. Continuation of the ESH-CHLSHOT trial after publication of the SPRINT: rationale for further study on blood pressure targets of antihypertensive treatment after stroke. J Hypertens. 2016;34:393-6.

15. Cushman WC, Evans GW, Byington RP, et al. Effects of intensive blood-pressure control in type 2 diabetes mellitus (ACCORD). NEJM. 2010;362:1575-85.

16. Xie X, Atkins E, Lv J, et al. Effects of intensive blood pressure lowering on cardiovascular and renal outcomes: updated systematic review and meta-analysis. Lancet. 2015;6736:1-9.

17. Cushman WC, Whelton PK, Fine LJ, et al. SPRINT Trial Results: Latest news in hypertension management. Hypertension. 2016;67:263-5.

18. Jamerson K, Weber MA, Bakris GL, et al. Benazepril plus amlodipine or hydrochlorothiazide for hypertension in high-risk patients. NEJM. 2008;359:2417-28.

19. Ettehad D, Emdin CA, Kiran A, et al. Blood pressure lowering for prevention of cardiovascular disease and death: a systematic review and meta-analysis. Lancet. 2016;387(10022):957-67.

20. Burroughs Peña MS, Mendes Abdala CV, Silva LC, et al. Usefulness for surveillance of hypertension prevalence studies in Latin America and the Caribbean: the past 10 years. Rev Panam Salud Publica. 2012;32:15-21.

21. Chow CK, Teo KK, Rangarajan S, et al. Prevalence, awareness, treatment, and control of hypertension in rural and urban communities in high-, middle-, and low-income countries. JAMA. 2013;310:959-68.

22. Miranda JJ, Herrera VM, Chirinos JA, et al. Major cardiovascular risk factors in Latin America: a comparison with the United States. The Latin American Consortium of Studies in Obesity (LASO). PLoS One. 2013;8:e54056.

23. Segura L, Agustín R, Ruiz E. La hipertensión arterial en el Perú según el estudio TORNASOL II. Rev Peru Cardiol. 2006;32:82-128.

24. Irazola VE, Gutierrez L, Bloomfield G, et al. Hypertension prevalence, awareness, treatment, and control in selected LMIC communities. Glob Heart. 2016;11:47-59.

25. Barquera S, Durazo-Arvizu R, Luke A, et al. Hypertension in Mexico and among Mexican Americans: prevalence and treatment patterns. J Hum Hypertens. 2008;22:617-26.

26. Shojania KG, Sampson M, Ansari MT, et al. How quickly do systematic reviews go out of date? A survival analysis. Ann Intern Med. 2007;147:224-33.

27. Shekelle PG, Ortiz E, Rhodes S, et al. Validity of the agency for healthcare research and quality clinical practice guidelines: how quickly do guidelines become outdated? JAMA. 2001;286:1461-7.

28. Alvarez-Vargas ML, Galvez-Olortegui JK, Galvez-Olortegui TV, et al. Clinical practice guidelines in hypertension: a review. Medwave. 2015;15:e6290.
29. James P, Oparil S, Carter BL, et al. 2014 evidence-based guideline for the management of high blood pressure in adults: report from the panel members appointed to the Eighth Joint National Committee (JNC 8). JAMA. 2014;311: 507-20.

30. Mancia G, Fagard R, Narkiewicz K, et al. 2013 ESH/ESC Practice Guidelines for the Management of Arterial Hypertension. Blood Press. 2014;23:3-16.

31. Daskalopoulou SS, Rabi DM, Zarnke KB, et al. The 2015 Canadian hypertension education program recommendations for blood pressure measurement, diagnosis, assessment of risk, prevention, and treatment of hypertension. Can J Cardiol. 2015;31:549-68.

32. Leung AA, Nerenberg K, Daskalopoulou SS, et al. Hypertension Canada's 2016 Canadian hypertension education program guidelines for blood pressure measurement, diagnosis, assessment of risk, prevention, and treatment of hypertension. Can J Cardiol. 2016;32:569-88.

33. Sforza VF. 2003 European Society of Hypertension-European Society of Cardiology guidelines for the management of arterial hypertension. J Hypertens. 2003;21:1011-53.

34. Dasgupta K, Quinn RR, Zarnke KB, et al. The 2014 Canadian Hypertension Education Program recommendations for blood pressure measurement, diagnosis, assessment of risk, prevention, and treatment of hypertension. Can J Cardiol. 2014;30:485-501.

35. Chobanian AV, Bakris GL, Black HR, et al. Seventh report of the Joint National committee on prevention, detection, evaluation, and treatment of high blood pressure. Hypertension. 2003;42:1206-52.

36. Alcocer L, Meaney E, Hernandez-Hernandez H. Applicability of the current hypertension guidelines in Latin America. Ther Adv Cardiovasc Dis. 2015;9:118-26.

37. Sanchez RA, Ayala M, Baglivo H, et al. Latin American guidelines on hypertension. J Hypertens. 2009;27:905-22.

38. López-Jaramillo P, Sánchez RA, Díaz M, et al. Consenso latinoamericano de hipertensión en pacientes con diabetes tipo 2 y síndrome metabólico. Clin Investig Arterioscler. 2013;26:85-103.

39. Guyatt G, Gutterman D, Baumann MH, et al. Grading strength of recommendations and quality of evidence in clinical guidelines: report from an american college of chest physicians task force. Chest. 2006;129:174-81.

40. Touyz RM, Dominiczak AF. Hypertension Guidelines Is it time to reappraise blood pressure thresholds and targets? Hypertension. 2016;67:688-9.

41. Galvez-Olortegui J, Camacho-Saavedra L, Godoy-Palomino A, et al. Changes in AGREE II assessment of CHEP Guidelines. Can J Cardiol. 2016. May 10 [Publicación electrónica].

42. National Institute for Health and Clinical Excellence. Hypertension in adults: diagnosis and management. London; 2011.

43. Arredondo A, Zuñiga A. Epidemiological changes and financial consequences of hypertension in Latin America: implications for the health system and patients in Mexico. Cad Saude Publica. 2012;28:497-502.

44. Gaziano TA, Bitton A, Anand S, et al. The global cost of nonoptimal blood pressure. J Hypertens. 2009;27:1472-7.

45. Escobedo-Palza S, Timana-Ruiz R, Sobrevilla-Ricci A, et al. Costo de hipertension arterial en los establecimientos de salud del Perú. Value Health. 2015;18:A829.

46. Dirección General de Medicamentos Insumos y Drogas. Observatorio de productos farmacéuticos [Internet]. Perú: www.minsa.gob.pe [consultado 17 Abr 2016]. Disponible en: http://observatorio.digemid.minsa.gob.pe/

47. Ramírez-Villaseñor I. Tratamiento farmacológico de la hipertensión arterial en medicina familiar: segunda parte. Arch en Med Fam. 2005;7:99-107. 
48. Gupta AK, Arshad S, Poulter NR. Compliance, safety, and effectiveness of fixed-dose combinations of antihypertensive agents: A meta-analysis. Hypertension. 2010;55:399-407.

49. Lemus F, Rivas R. Affordability of antihypertensive treatment in Mexico. Value Health. 2013;16:A705.

50. Kulkarni SP, Alexander KP, Lytle B, et al. Long-term adherence with cardiovascular drug regimens. Am Heart J. 2006;151:185-91.
51. Bittner M, Espinoza MA, Montero J. Evaluación de costoefectividad de la implementación de monitoreo ambulatorio de presión arterial versus perfil de presión arterial para el diagnóstico de hipertensión en el sistema público chileno. Value Health. 2015;18:A859.

52. Moran AE, Odden MC, Thanataveerat A, et al. Cost-effectiveness of hypertension therapy according to 2014 guidelines. NEJM. 2015;372:447-55. 